\title{
LAS ESTRUCTURAS DEL PAISAJE VEGETAL DEL PIRINEO OCCIDENTAL Y SU ESTABILIDAD*
}

\author{
LUis VILlaR**
}

\section{RESUMEN}

Se describe el mosaico de estructura del paisaje vegetal del Pirineo occidental sobre la base de datos geológicos, climáticos, de relieve y de explotación natural y humana. Se comenta su estabilidad a lo largo de los siglos en un sistema de civilización ganadera y silvo-pastoral. Sin embargo, en los últimos tiempos, la explotación indiscriminada provoca la erosión del suelo, amenazando con producir una fuerte simplificación del tapiz vegetal mediante procesos irreversibles.

\section{RÉSUMÉ}

L'auteur envisage le mosaïque structural du paysage végétal des Pyrénées Occidentales espagnoles sur la base des données géologiques, climatiques et du relief. Il tient aussi compte de l'exploitation naturelle et humaine. La stabilité de ces paysages, maintenue au long des siècles au sein díune civilisation pastorale et silvo-pastorale, est étudiée. Cependant, au cours de nos jours, une exploitation trop intense, au moyen de l'érosion du sol, nous mène à une forte simplification du manteau végétal.

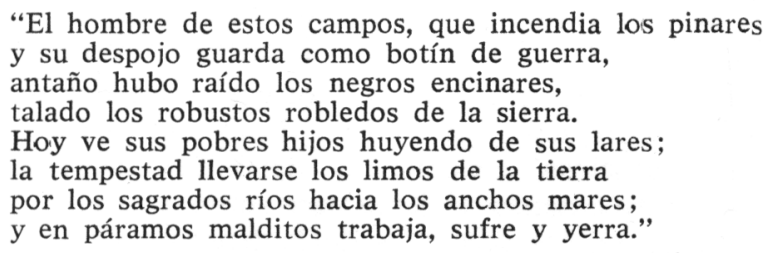

\section{INTRODUCCIÓN}

Quizá como reacción a las teorías homogenistas de la clímax, desde hace algunos años se viene comprobando que las estructuras heterogéneas predominan de un modo general en la naturaleza. Estructuras en mosaico se pueden observar desde el nivel físico al paisajístico, pasando por el macromolecular, celular, histológico, ecológico, etc.

Hace también poco tiempo que se han asimilado estas estructuras reticulares a la teoría ecológica general relacionándolas con el concepto

$\left(^{*}\right)$ Comunicación leída en el Seminario sobre Estructura y Estabilidad del Ecosistema, Sevilla, noviembre de 1974.

(**) Centro Pirenaico de Biología Experimental. Apartado 64. IACA (Huesca). 
de estabilidad. Parecen ser, en efecto, muy estables en el tiempo y se ha recomendado su uso para organizar el paisaje con fines de explotación y conservación (MARGALEF, 1970).

Anteriormente habíamos esbozado la idea de mosaico al paisaje vegetal de un valle del Pirineo occidental (VIllar, 1973). Luego Dendaletche (1973) habló de la malla constituida por estructuras geológicas longitudinales y red fluvial transversal.

Pretendemos ahora ampliar estas ideas a todo el Pirineo occidental en relación con la estabilidad (entendida esta como la persistencia de un sistema en el tiempo así como su capacidad de retorno a una situación de equilibrio, cuando se ha separado de ella) y busca fundamentos ecológicos generalizables a todas las zonas de relieve montañoso.

\section{LÍMITES Y RASGOS GEOGRÁFICOS}

El Pirineo occidental español, tal como aquí lo entendemos (MonTseRRAT, 1974a), ocupa parte del Alto Aragón occidental y de la Navarra oriental. Se halla limitado al este por el Valle de Canfranc y al oeste por el Valle del Roncal, ambos de dirección meridina. La divisoria de aguas entre ambas vertientes constituye su límite septentrional y la Depresión Altoaragonesa o Canal de Berdún el borde natural meridional, más allá del cual se encuentran las Sierras Prepirenaicas.

Geología, clima y relieve independizan esta zona de los vecinos Pirineos Centrales (Valle de Tena, contiguo) y también del Pirineo Atlántico, situado a poniente.

Sus altitudes oscilan entre los 600-1.000 m de Depresión Media y los 2.668 del Bisaurín en las Sierras Interiores calizas, que con frecuencia sobrepasan los $2.300 \mathrm{~m}$ (Anie, Lacherito, Forca, Agüerri, Aspes...)

$\mathrm{Su}$ estructura geológica nos ofrece montañas de dirección aproximada este-oeste formadas por calizas duras en varias bandas paralelas. Estas calizas cretácicas, eocénicas y devónicas presentan un relieve kárstico a veces muy extenso y descansan sobre rocas no básicas del Permotrías, que afloran en las cercanías de la frontera franco-española. Los terrenos, mucho más flexibles del flysh eocénico, dominan al sur y oeste de las calizas abruptas.

La red hidrográfica es de sentido fundamental, meridiano de suerte que todos los ríos han formado valles que desde el norte descienden a la Canal de Berdún. Existen algunos valles laterales, más o menos transversales, los cuales son más largos y amplios en las márgenes izquierdas de los ríos principales. (Véase mapa).

La parte alta de los valles suele ser amplia por circunstancias tectónicas, mientras que la parte media presenta una sucesión en "rosario" de porciones amplias, debidas a sustrato blando fácilmente erosionable y porciones estrechas, en forma de profundos desfiladeros o "foces", excavados en las calizas duras mencionadas.

Como rasgo climático dominante, las masas de aire oceánico progresan en sentido noroeste-sureste, bañando las vertientes umbrías y provocando 
en las solanas efecto foehn. Esta influencia oceánica se traduce en altitud en una innivación intensa y prolongada.

Por el contrario, desde la parte sudoriental alcanzan las estribaciones pirenaicas los efluvios mediterráneos-continentales. La torrencialidad se incrementa de Navarra a Aragón. Estos fenómenos permiten definir un gradiente señalado oceanidad-continentalidad, de oeste a este, y atlanticidad-mediterraneidad, de noroeste a suroeste. El anastomosamiento de tales influencias sobre un relieve montañoso provoca un mosaico abigarrado de topoclimas.

Los suelos son fundamentalmente básicos, pero las circunstancias climáticas mencionadas provocan fenómenos de lavado en la parte occidental, hacia Navarra, con lo que se acentúa la acidez. La continentalidad oriental hace que sea la erosión mecánica predominante hacia este lado.

Mención especial merecen los suelos espesos, probablemente fósiles, situados entre los 1.600-2.300 m de altitud, sobre flysch maestrichtiense, ahora en proceso de erosión debido seguramente a un desequilibrio con el clima actual (Negre, Dendaletche y Villar, 1975).

Los glaciares dejaron escasas huellas entre las Sierras de Aísa y el Macizo del Anie, pero, sin embargo, persisten actualmente, con cierta intensidad, los fenómenos periglaciares de crioturbación y solifluxión.

Relieve estructural dirigido fundamentalmente de este a oeste, relieve erosivo dirigido de norte a sur, gran desarrollo del karst y del flysch, suelos básicos, suelos fósiles, clima de contacto entre lo atlántico y lo mediterráneo-continental, he aquí los principales rasgos del medio físico en el Pirineo Occidental español.

\section{EL PIRINEO OCCIDENTAL Y SU PAISAJE VEGETAL}

El tapiz vegetal de la región que acabamos de esbozar reacciona de modo muy aparente a las condiciones ecológicas mencionadas.

Para su representación esquemática nos basaremos en la descripción y cartografía realizada por MonTserRat en su libro La acetania y su vida vegetal (1971).

A grosso modo podemos dividir la vegetación de estas montañas en dos grandes conjuntos: el complejo de los bosques, comprendido entre los 800-1.800 $(2.000 \mathrm{~m})$ y el complejo supraforestal, entre los (1.800) 2.000$2.600 \mathrm{~m}$ de altitud.

De abajo arriba, la banda forestal nos presenta la siguiente zonación altitudinal :

Áreas de los robledales submediterráneos, de hoja marcescente o "quejigales" con boj, gayuba, etc..., algo mezclados con pino albar. Por la zona de poniente la erosión química se destaca por las plantas subcantábrica de brezal. En los lugares donde la evaporación es intensa, el suelo esquelético y la insolación fuerte se presentan islotes con encinas o plantas mediterráneas. Ocupan todas las partes bajas de los valles principales 
y penetran por sus laderas inferiores y medias hasta muy adentro, en suelos relativamente profundos.

Área de los pinares musgosos: Las masas de pino albar $(P$. silvestris) con estrato muscinal ocupan buena parte de las estructuras transversales del flysch en distintas exposiciones, excepto en algunas cresterías dominadas por matorrales de boj y erizón. Alcanza los 1.500-1.600 m.

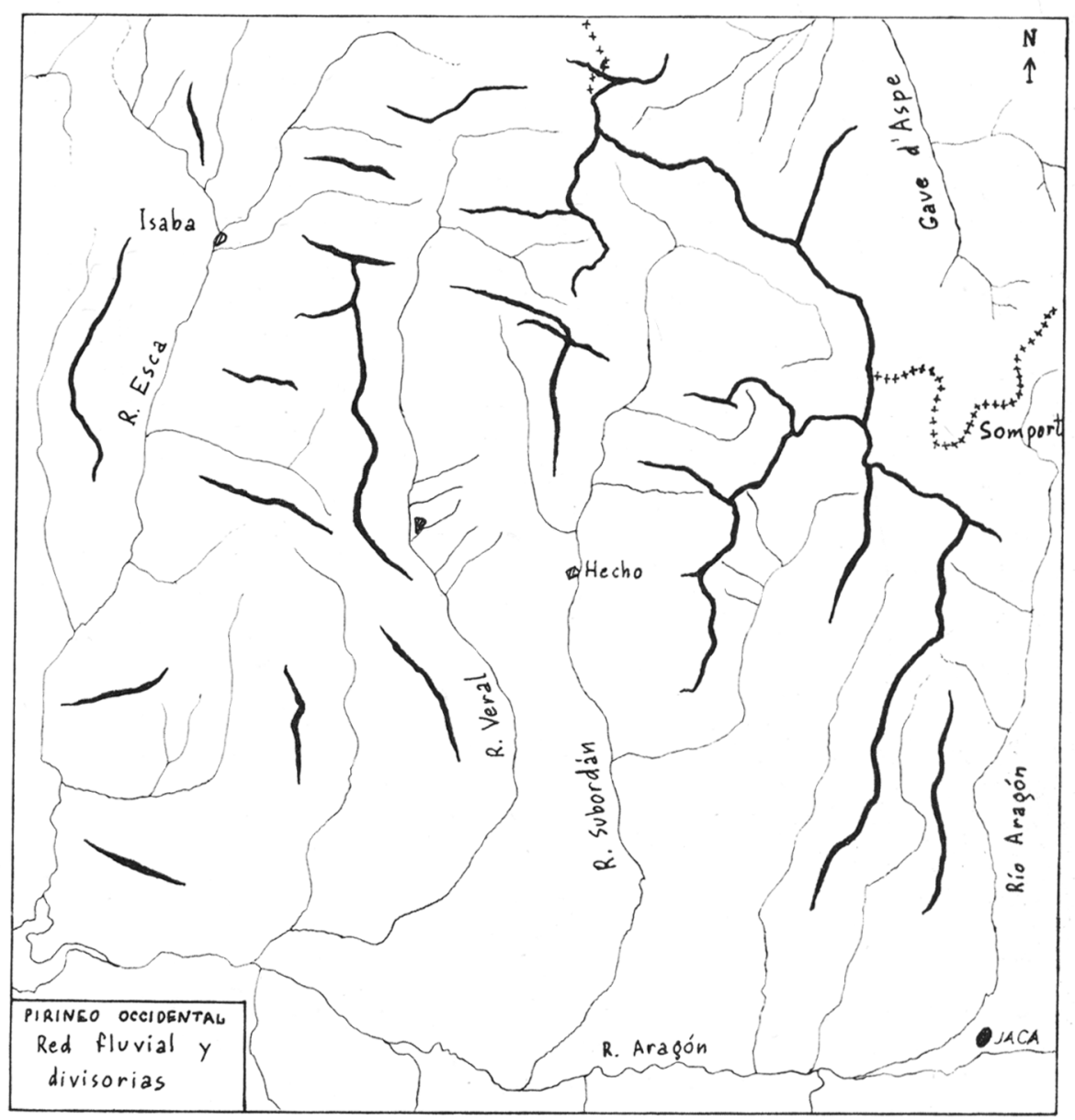

Area de los hayedos con abeto o de los abetales con haya: Dominan en la parte nor-occidental y norte de la zona estudiada, asentándose los hayedos en las zonas bañadas por las nieblas y los abetales en suelos profundos y húmedos de pendiente suave o en fondos de valle. Altitudes entre los 1.000-1.600 m.

Area de las coniferas del límite superior forestal: Los pinares de pino negro, ya sea con toda la cohorte del matorral subalpino a base de 
azaleas y arándanos, ya sea con un estrato herbáceo de Festuca scoparia de afinidad oromediterránea, se enseñorean de la zona entre los (1.500) $1.600-2.000 \mathrm{~m}$, salpicando en numerosos islotes los abruptos ralos las grandes extensiones kársticas.

Piso supraforestal dominado por los pastizales de Festuca scoparia que forman grandes manchas en las solanas de los altos valles de AnsóHecho-Canfranc y cervunales ansotanos en los lugares de mayor innivación o abiertos a las cantábricas. Encontramos, por último, los pastos alpinos de la clase Elyno-Seslerietea y las grandes extensiones minerales kársticas con vegetación fisurícola, glareícola y de las crestas venteadas.

En conjunto, a esta escala del paisaje vegetal, el mapa citado presenta 17 unidades distintas. Una corresponde a la vegetación mediterránea: "Madroñales con Viburnun tinus en gargantas húmedas y cálidas". Cuatro, a la vegetación submediterránea: "Carrascales montanos con boj y gayuba", "Enclaves cálidos; refugio de plantas termófilas", "Quejigales secos jacetanos y prepirenaicos con sus etapas seriales" y "Quejigales secos jacetanos y prepirenaicos con sus etapas seriales" y "Quejigales navarros con suelo acidificado superficialmente, algunos brezos, Cistus salviifolius y Thymelaea ruizii". Tres, al piso montano inferior seco: "Pinar con boj y frecuentemente Genista horrida", "Crestones secos y ventosos con erizón, Genista horrida dominante" y "Pinar musgoso con boj”. Cuatro, al piso montano inferior húmedo: "Hayedos", "Abetales con haya”, "Robledales y prados de guadaña frescos del fondo plano de los grandes valles pirenaicos y al pie de los grandes cantiles" y "Olmedas de las riberas, huertas, prados, choperas, juncales y salguerales. Bosques muy húmedos en los montes". Tres, a la alta montaña mediterránea alpina: "Complejo del pino negro con Festuca scoparia y bufalagas", "Piso subalpino con rododendro y arándanos. Pirineo Central" y "Pastos secos y soleados con Festuca scoparia y algunas veces Festuca eskia". Dos, finalmente, al piso alpino o alpinizado: "Complejo del cervunal ansotano brumoso y sobre suelos pobres en cal o decalcificados" y "Complejo del pasto seco sobre roca caliza; subalpino y alpino".

\section{LAS ESTRUCTURAS RETICULARES DEL PAISAJE VEGETAL Y SU FUNDAMENTO}

Las distintas unidades del paisaje del Pirineo Occidental expresan un ejemplo claro de las estructuras reticulares. Ya ocurría así antes de que la influencia humana se generalizase; esta última no habría hecho sino acrecentar, desmenuzar o alterar el mosaico preexistente.

En las líneas que siguen pretendemos buscar las causas de estas estructuras en mosaico y de su estabilidad a lo largo del tiempo. Trataremos el caso a través de la explotación natural biótica y abiótica, puesto que sin duda es uno de los mecanismos naturales de rejuvenecimiento de comunidades; o sea, de creación de estructuras en red. He aquí las causas diversificadoras que mencionamos: en relieve, acontecimientos biogeográficos, naturaleza del sustrato y explotación animal-humana. 


\section{EL RELIEVE Y LAS ESTRUCTURAS EN MOSAICO}

El establecimiento de las comunidades vegetales de distintas afinidades ecológicas o de distintos grados de madurez viene condicionado, en las zonas montañosas, sobre todo por el relieve. Los factores oroclimáticos explotan a determinadas comunidades sustrayéndoles materia o desviando la energía que podría organizarlas (VILlaR, 1975). La acumulación de fertilidad en el suelo y las etapas de la sucesión creciente se ven limitadas en los siguientes microhábitats: 1) laderas pendientes; 2) crestas venteadas; 3) lugares sometidos a aludes o desprendimientos; 4) espolones azotados por los rayos; 5) pedrizas; 6) cantiles y grietas; 7) lugares sometidos a fenómenos periglaciares; 8) extensiones kársticas; 9) ventisqueros, y 10) barrancos, arroyos o lechos de río.

En el Pirineo Occidental, donde la vegetación eurosiberiana entra en contacto con la mediterránea, donde el clima atlántico y el mediterráneo hacen otro tanto, se comprende que simplemente la exposición determine el predominio de una u otra unidad vegetal o que los barrancos umbríos, con una elevada higrometría y aporte continuo de fertilidad desde las laderas, posean las selvas más maduras y estructuradas. La explotación natural abiótica (MONTSERRAT y VILlaR, 1972), propia de cualquier relieve montañoso, implica la existencia de una serie de comunidades simplificadas, permanentes $o$, si se quiere, explotadas, frente a otras dotadas de mayor biomasa.

\section{FONDO FLORAL, ACONTECIMIENTOS BIOGRÁFICOS Y ESTRUCTURAS EN MOSAICO}

Sabemos que en la Era Terciaria, el largo período Mioceno permitió la llegada de la vegetación esteparia a los Pirineos. Plantas iberomauritanas de este origen se instalaron en las vertientes meridionales soleadas cuyo clima las favorecía y donde se han mantenido hasta hoy. (Una prueba geomorfológica de que las condiciones miocénicas alcanzaron los Pirineos es la existencia de relieves maduros "colgados" en los valles medios de Roncal y Salazar: Planta de Sasi y Coronas de Navascués. Cf. Barrere, 1962). Las glaciaciones cuaternarias, aunque provocaron vaivenes de la flora y de la vegetación, verosímilmente no alteraron estos lugares ni hicieron desaparecer totalmente la vida vegetal de las altas cumbres (nunataks). (Es un hecho geobotánico general que las vertientes meridionales de los Pirineos y los Alpes han tenido un papel conservador de flora terciaria). Unos y otros islotes, junto con los colonizadores más recientes de las áreas glaciadas, contribuyen a las estructuras heterogéneas actuales.

\section{LA NATURALEZA DEL SUSTRATO Y EL MOSAICO}

Aunque algunas especies son indiferentes a la naturaleza de la roca madre, la diversidad del sustrato aumenta también la diversidad del tapiz vegetal. Así, por ejemplo, los piornales de Cytisus purgans y Genista florida forman unidades aisladas en suelos permotriásicos y no se encuentran jamás en los suelos calizos predominantes en el Pirineo Occidental.

Enumeraremos a continuación las catorce unidades que componen el mosaico de facies litológicas del Pirineo Occidental (Soler y PuigdefáBREGAS, 1972): 1) Aluviones y glacis en general calcáreos; 2) Depósitos de morrenas glaciares; 3) Depósitos de conos de deyección; 4) Depósitos 
de ladera, canchales, etc.; 5) Conglomerados y areniscas silíceas; 6) Areniscas y arcillas en facies Permotrías; 7) Arcillas rojas del Permotrías; 8) Arcillas, areniscas y calizas lacustres: Garumnense; 9) Margas alternando con areniscas en bancos delgados: flysch arenoso; 10) Calizas marinas masivas; 11) Calizas alternando con areniscas y margas; 12) Pizarras arcillosas alternando con areniscas; 13) Grauvacas alternando con pizarras; y 14) Rocas efusivas básicas.

Las calizas masivas y las margas del flysch son las que predominan.

\section{LA EXPLOTACIÓN NATURAL BIÓTICA COMO ACRECENTADORA DEL MOSAICO}

Como hemos visto, las oscilaciones climáticas en el espacio y en el tiempo han contribuido en otras épocas a la formación de mosaicos de estructura vegetal, algunos de cuyos elementos persisten en la actualidad. Pero clima y suelo no bastan para explicarnos las estructuras diversificadas de nuestro paisaje. Hemos de acudir a los animales y al hombre para echar luz al asunto.

Por su posición en la red trófica, la biomasa animal rejuvenece a los vegetales acelerando el flujo energético que atraviesa los productores primarios. Tal rejuvenecimiento, diferenciado en el espacio, da lugar a una modificación de las estructuras ya existentes, bien sea alterando sus proporciones o bien creando otros mosaicos a escala menor.

Generalizando, la acción de los animales sobre la vegetación se ha materializado en una simplificación creciente de las estructuras forestales. Los grandes herbívoros tenían y tienen querencias forestales desarrolladas seguramente a partir de los primeros claros naturales del bosque, que se fueron ensanchando.

No obstante, al menos en épocas postglaciares, la densidad de los herbívoros salvajes ha sido discreta - controlada quizá por los abundantes consumidores: lobo, oso y hombre, principalmente- produciendo una suave simplificación del complejo y denso manto forestal de quejigos, encinas, hayas, abetos, pinos, tejos, olmos, avellanos, etc., así como un incremento moderado de las orlas del bosque a base de arbustos de crecimiento algo más rápido.

Pero esta situación de equilibrio favorable a la biomasa vegetal cambió cuando en los tiempos prehistóricos el hombre pasó de ser frugívoro y cazador a pastor: domesticó a los herbívoros y empezó a desarrollar prácticas favorables al pastoreo. Con ello el proceso de vaciado de los bosques pirenaicos se aceleró. Los pequeños claros forestales se transformaron en extensas manchas de pastizales gracias al sobrepastoreo y al fuego. Asimismo grandes áreas forestadas cerca del límite forestal-supraforestal se vieron reducidas a comunidades herbáceas (Piso subalpino alpinizado): el límite superior del bosque descendió.

Con la civilización pastoral se llegó a un nivel de simplificación mayor, aunque continuaron persistiendo manchas de selvas vírgenes más o menos originarias.

Esta época ha durado hasta el siglo xviII y estaba caracterizada por una gran diversidad de ganados domésticos, una mínima explotación di- 
recta de las comunidades forestales por parte del hombre (con poblaciones más o menos constantes salvo en las guerras) y, en conjunto, un buen nivel de estabilidad. Tan solo la erosión de las pequeñas parcelas agrícolas de los valles medios y bajos o de las cercanías de los núcleos habitados rompía el equilibrio.

Se trataba de un sistema casi cerrado salvo en lo referente a la trashumancia obligada, un sistema bastante controlado por las poblaciones humanas autóctonas. Era un sistema equilibrado.

Con la civilización industrial, las grandes obras públicas y las crecientes necesidades de la Armada, el control de los bosques pirenaicos pasó de los montañeses a nivel nacional y ello produjo una simplificación mayor. La exportación de madera al exterior no dejó de acrecentarse hasta la actualidad.

Así los bosques han dejado de ser parcelas más o menos homogéneas y se han transformado en zonas reticulares donde la proporción de los diversos elementos del mosaico a la superficie total se ha alterado sustancialmente. Los bosques maduros o inalterados constituyen verdaderas islas o más bien quedan solo islotes; la fauna que albergaban se ha ido enrareciendo o extinguiendo acosada por el hombre. Los pinares secundarios han sustituido a los hayedos.

La simplificación debida a explotaciones poco cuidadosas y al clima ha alcanzado el subsistema suelo: la erosión ha comenzado.

\section{LA ESTABILIDAD DEL PAISAJE VEGETAL EN EL PIRINEO OCCIDENTAL}

Desde la Prehistoria, el manto forestal casi continuo de los pisos submediterráneo, montano y subalpino pirenaicos se ha visto sometido a un proceso de aclareo que aumentó sus discontinuidades. Las complejas comunidades maduras formaban áreas continuas muy extensas dentro de las cuales había pequeñas manchas de comunidades más simples (pastos y matorrales), por explotación natural del clima, el relieve, los animales y el hombre.

En el piso supraforestal siempre hubo heterogeneidad, debida sobre todo a la explotación abiótica.

Ya en nuestra época histórica, mientras el control de la explotación tenía lugar a nivel local (Prehistoria) o regional (civilización ganadera trashumante) no tuvieron lugar procesos irreversibles en el tapiz vegetal, excepto pequeñas heridas agrícolas. La malla o mosaico estructural de escasas superficies aradas, bosques claros con pastos y setos, muchos bosques densos y grandes extensiones pastorales supraforestales daba una producción exportable sostenida: productos agrícolas, leña, ramas tiernas de árboles, madera para edificaciones locales, animales domesticados de todas clases, caza y pesca. El suelo permanecía inalterado salvo en aquellos lugares sometidos a fuerte explotación abiótica.

La escasa erosión física producida por tormentas, rayos, incendios y aguas torrenciales era pronto absorbida un poco más abajo por la "esponja" de bosques densos y pastos bien encespedados. La fertilidad no descendía 
aguas abajo. Los ríos tenían escasas avenidas, aguas muy claras y el delta del Ebro recibía muy pocos aportes pirenaicos occidentales.

Pero al pasar a nivel nacional o internacional, el sistema se convirtió en subsistema explotado de otro sistema mucho mayor. El flujo de energía que lo atravesaba se aceleró. La madera descendió en grandes masas, transportada primero por los ríos, luego por las carreteras. Parcelas forestales maduras que nunca habían "experimentado" la explotación, sufrieron talas intensas.

Empezó a desencadenarse un proceso irreversible: la erosión del suelo. La mencionada “esponja” forestal se convirtió en cedazo y por sus agujeros filtraron las tierras fértiles y su energía potencial. Las aguas desatadas las transportaron hasta los embalses o el mar, contribuyendo a su eutrofización y colmatación.

Con la simplificación general provocada por el mercado internacional de carne, madera y "bulldozers", así como con la erosión subsiguiente del suelo estamos tocando el fondo, llegando al límite de la flexibilidad que tienen las comunidades vegetales para volver a su estado primitivo. El ecosistema montaraz se desmorona, sufre grandes trastornos irreversibles. Estamos haciéndole perder su estabilidad milenaria.

\section{EXPLOTACIÓN, ESTABILIDAD Y CONSERVACIÓN : UNA ESTRATEGIA A SEGUIR}

En ecosistemas montañosos muy diversificados, de rica estructura, como son los montañosos, el grado de explotación tiene que ser también diversificado para cada elemento de su mosaico.

Los pastos son comunidades dispuestas a dar una alta producción exportable de un modo continuo, sin desorganizarse. En realidad ya estaban preadaptadas para ello por su elevada tasa de renovación.

En cambio, el "turnover" de los bosques es mucho menor y por ello su producción de biomasa exportable más baja. En el Pirineo Occidental los más productivos son los de pino albar, luego los buenos abetales, los hayedos y por último los pinares de pino negro.

La mayor parte de las explotaciones sobre bosques y pastos son reversibles a corto y medio plazo a condición de que no se degrade el suelo, ya que su lento proceso de rehabilitación se sale de nuestra escala histórica.

Pero en los climas de influencia oceánica se ceba la erosión química cuando los planifolios (hayas) son sustituidos por aciculifolios (pinos). Y la erosión mecánica progresa en las laderas y pendientes donde el bosque se explota demasiado o en donde, aún siendo menor la pendiente, los procedimientos de arrastre y "saca” de madera excavan el suelo.

Hablando con el símil económico, el suelo es el "capital” de cualquier negocio biológico terrestre. Si lo dejamos perder nos estamos "descapitalizando". No solo simplificaremos rápidamente los ecosistemas, sino que comprometeremos cualquier posible utilización futura.

Nuestra principal estrategia para mantener la estabilidad de las estructuras vegetales del medio terrestre debe ser la conservación del suelo. 


\section{CONCLUSIONES}

1. En la Naturaleza dominan las estructuras reticulares, heterogéneas o en mosaico a todos los niveles, desde el celular hasta el ecológico y paisajístico.

2. Tales estructuras en mosaico son muy aparentes en las zonas de relieve montañoso como el Pirineo Occidental, sobre todo en el paisaje vegetal.

3. Clima, relieve, historia biogeográfica, litología y animales (incluido el hombre) son los principales factores que condicionan las estructuras en mosaico del tapiz vegetal del Pirineo Occidental.

4. El paisaje silvo-pastoral de bosques densos con algún claro encespedado, prados supraforestales y alpinos se ha revelado muy estable ante la explotación de los animales salvajes e incluso ante la milenaria civilización pastoral humana.

5. Herbívoros salvajes, domésticos y hombre han provocado un proceso de simplificación creciente de las estructuras forestales y vegetales en general. Tal proceso se mantuvo dentro de límites estables para el sistema hasta el siglo XVIII, momento en que se intensificó considerablemente la explotación forestal.

6. Esta explotación forestal acrecentó en gran escala los procesos de erosión del suelo que por su carácter irreversible amenazan con degradar todo el ecosistema montaraz del Pirineo Occidental.

7. Para mantener la estabilidad de las estructuras vegetales del Pirineo Occidental debemos evitar la erosión. El subsistema edáfico es el que mayor estabilidad proporciona a los ecosistemas terrestres.

8. Si la erosión continúa, solo supervivirán en el futuro, seguramente, las comunidades explotadas, dado que están "preadaptadas" a los suelos simplificados.

9. Según el principio general "La heterogeneidad en el espacio produce homogeneidad o estabilidad en el tiempo mientras que la uniformidad espacial provoca inestabilidad temporal" (MARGalef, com. verb.) podemos concluir que las estructuras naturales en mosaico provocan cierta penetración en el tiempo por parte de los ecosistemas.

Agradecimiento: El autor agradece al Dr. P. MonTSERRAt la corrección del manuscrito y al Dr. R. MARGAlef su sugerencia respecto a la conclusión 9.

\section{BIBLIOGRAFIA}

Barrere, P., 1962. "Reliefs mûrs perchés de la Navarre orientale". Revue Géorg. Pyr. et du Sud-Ouest, 33(4): 309-323. Toulouse.

Berducou, C., 1974. "Contribution á l'étude d'un probléme écophysiologique pyrénéen: L'alimentation hivernale de l'Isard". Thèse. Université Paul Sabatier. Toulouse.

Bolos, O. DE, 1963. "Botánica y Geografía”. Mem. Real Ac. Ciencias y Artes de Barcelona, 34(14): 443-491. Barcelona.

Bolos, O. DE, 1973: "La conservación de la Naturaleza y sus fundamentos". Consejo Superior de Investigaciones Científicas. Barcelona. 
Bolos, O. De y Montserrat, P., 1960. "Guide de la partie espaghole". Excursión de l'Association Internationale de Phytosociologie dans les Pyrénées Centrales et Occidentales, 15 pp. mecanografiadas.

Dendaletche, C., 1973. "Ecologie et peuplement végétal des Pyrénées Occidentales. Essai d'écologie montagnarde". These. Université de Nantes. Cf. Bull. Cent. Étud. Rech. Sci. Biarritz, 9(2): 141-207 (1972). Biarritz.

Margalef, R., 1970. "Estructuras del paisaje vegetal más apropiadas a la misión de conservación". En "Simposio de Conservación de la Biosfera". Revista de Geografía. Barcelona.

MontSerrat, P., 1971. "La Jacetania y su vida vegetal", 108 pp. y mapa en color. Zaragoza. Véase también "El ambiente vegetal jacetano". Pirineos, 101: 5-22, con mapa en color. Jaca.

Montserrat, P., 1971. "El clima subcantábrico en el Pirineo Occidental español". Pirineos, 102: 5-19. Jaca.

Montserrat, P., 1972. "Estructura del sistema agropecuario". Anales de Edafología y Agrobiologia, 31(1-2). Madrid.

Montserrat, P., 1974. "L'exploration floristique des Pyrénées Occidentales". Bol. da Soc. Broteriana, 47(2. ${ }^{\mathrm{a}}$ ser.), suplemento: 227-240. Coimbra.

Montserrat, P., 1974b. "Continentalidades climáticas pirenaicas". Comunicación presentada en el VII Congreso Internacional de Estudios Pirenaicos. Seo de Urgel, septiembre de 1974. En prensa.

MontSerrat, P., 1974c. "Clima y paisaje". Comunicación presentada a la IV Reunión de Climatología del Patronato Alonso de Herrera. P. Cent. Pir. Biol. Exp., 7. Jaca.

MontSerrat, P., 1974d. "Estructura y estabilidad de los Ecosistemas. Relaciones con la utilización de los recursos". Ponencia leída en el Seminario sobre Estructura y Estabilidad del Ecosistema. Sevilla, noviembre de 1974. En prensa.

Montserrat, P. y Villar, L., 1972. "El endemismo ibérico: aspectos ecológicos y fitotopográficos”. Bol. Soc. Broteriana, 46: 503-527. Coimbra.

Negre, R., Dendaletche, C. y Villar, L., 1975. "Les groupements á Festuca paniculata en Pyrénées Centrales et Occidentales”. Bol. Soc. Broteriana, 49. Coimbra.

Puigdefábregas, J. y Balcells, E., 1970. "Relaciones entre la organización social y la explotación del territorio en el Valle del Roncal (Navarra oriental)". Pirineos, 98: 53-90. Jaca.

Soler, M. y Puigdefábregas, C., 1972. "Esquema litológico del Alto Aragón occidental". Pirineos, 106: 5-15, con mapa en color. Jaca.

Villar, L., 1973.“Explotación y Conservación de la Naturaleza en el Alto Roncal”. P. Inst. Biol. Aplicada, 54: 129-148. Barcelona.

Villar, L., 1975. "El clima como agente de explotación natural". Comunicación presentada a la IV Reunión de Climatología del Patronato Alonso de Herrera. P. Cent. Pir. Biol. Exp., 7. Jaca.

Villar, L. y García-Ruiz, J. M., 1974. "Explotación del territorio y evolución de pastos en dos valles del Pirineo Occidental". Pirineos, 112. Jaca. 